\title{
Impact of Computerized Provider Order Entry Systems on hospital staff pharmacist workflow productivity: A three site comparative analysis based on level of CPOE implementation
}

\author{
Benjamin D. Lewing, Mark D. Hatfield, Sujit S. Sansgiry* \\ Department of Pharmaceutical Health Outcomes and Policy, University of Houston, Houston, United States
}

Received: October 6, 2017

Accepted: December 8, 2017

Online Published: December 22, 2017

DOI: $10.5430 /$ jha.v7n1p1

URL: https://doi.org/10.5430/jha.v7n1p1

\begin{abstract}
Objective: Computerized Provider Order Entry (CPOE) is a system that enables physicians to send medication orders electronically rather than physically writing out the order. CPOE can reduce handwriting and transcription related medication errors and has been a major implementation goal for health systems. The objective of this study was to quantify and examine differences seen in the workflow of pharmacists at hospitals, with different levels of CPOE implementation.

Methods: An observational, prospective time and motion study was conducted among three hospitals within the same health system: one classified as a non-CPOE system, one as short-term CPOE, and one as long-term CPOE. Pharmacists were observed in one-hour blocks, in which a data instrument was used to record 38 different tasks, which were grouped into four activities: clinical, distributive, administrative, and miscellaneous. The distributive category was further divided into three sub-categories. The average time associated with performing activities across the three hospitals was compared by descriptive and comparative analyses using ANOVAs and the post-hoc Tukey's range test.

Results: A total of 252 hours were collected and 235 met the inclusion criteria. The significant differences in time spent on task categories among hospitals were as follows: Non-CPOE vs. short-term CPOE vs. long-term CPOE (mean $\pm S D$ in min/h) clinical tasks: $(6.55 \pm 6.40)$ vs. $(4.95 \pm 4.15)$ vs. $(3.79 \pm 4.91)$, respectively, $(p<.05)$; order entry tasks: $(29.62 \pm 11.24)$ vs. (17.44 \pm $10.73)$ vs. $(10.27 \pm 8.88)$ respectively, $(p<.05)$; order verification tasks: $(0.88 \pm 1.77)$ vs. $(13.93 \pm 8.50)$ vs. $(16.60 \pm 9.63)$ respectively, $(p<.05)$; other distributive tasks: $(13.60 \pm 10.04)$ vs. $(15.86 \pm 8.38)$ vs. $(19.66 \pm 8.42)$ respectively, $(p<.05)$; and miscellaneous: $(3.78 \pm 4.64)$ vs. $(1.54 \pm 3.20)$ vs. $(2.23 \pm 3.51)$ respectively, $(p<.05)$.

Conclusions: The presence of a CPOE system could affect pharmacists' workflow and time allotment on different types of pharmacy activities. Further, the time spent on certain activities was associated with the amount of time the CPOE system was implemented.
\end{abstract}

Key Words: Computerized Provider Order Entry, Pharmacist productivity, System implementation, Hospital pharmacy workflow, Time-and-motion

\footnotetext{
*Correspondence: Sujit S. Sansgiry; Email: ssansgiry@uh.edu; Address: Health and Biomedical Sciences Building 2, Room 4055. 4849 Calhoun, College of Pharmacy, Houston, United States.
} 


\section{INTRODUCTION}

Computerized Provider Order Entry (CPOE) is a system that allows physicians to provide medication orders electronically rather than manually writing the order, thereby helping to reduce handwriting or transcription related medication errors. ${ }^{[1]}$ Implementation of CPOE can significantly reduce medication errors which can lead to adverse drug events. ${ }^{[2-4]}$ In addition, implementation or improvement of CPOE in hospitals was a major goal for organizations across the US, including the Leapfrog Group, Centers for Medicare and Medicaid (CMS) and the Agency for Healthcare Research and Quality (AHRQ). ${ }^{[5-7]}$

Adoption of CPOE is part of Stage Two of the Meaningful Use Program of the incorporation of health information technology into hospitals, part of the CMS Electronic Health Record Incentive Programs. ${ }^{[7]}$ In 2014, the Office of the National Coordinator for Health Information Technology published a report indicating the percentage of US hospitals considered to have adopted meaningful use of CPOE for medications, which rose from $69.8 \%$ in 2012 to $84.4 \%$ in 2013. ${ }^{[8]}$

While CPOE systems have been recognized for their valuable attributes including tools to reduce cost, increase efficiency, and reduce medication errors, they have also been found to have numerous barriers of use. One particular barrier is a shift in workflow. ${ }^{[9]}$ Published studies regarding pharmacist workflow change following implementation of $\mathrm{CPOE}$ have shown varying and conflicting results. ${ }^{[10-18]}$ A literature review of 51 publications regarding the implementation of CPOE found that two articles indicated a decrease in interruptions due to clarifying ineligible orders after implementing a CPOE system, and six studies indicated turnaround time on drug orders was significantly decreased. ${ }^{[10]}$ However, several studies reported workflow interruptions including CPOE system availability, such as system malfunction or system overload during peak times, and numerous issues with human-computer interactions. ${ }^{[13,15-18]}$ Further, while one study indicated a decrease in the time spent by pharmacists on the medication process after CPOE implementation, another study indicated no difference. ${ }^{[10,11,16]}$

With the significance placed on hospital usage of CPOE and the rapid rate in which CPOE is being adopted, pharmacy leadership will be forced to examine how the incorporation of CPOE affects workflow. It has been previously reported that CPOE would allow pharmacists to expand their role and spend more time on clinically related activities. ${ }^{[19]}$ In an article published previously, implementation of CPOE was shown to increase pharmacist time spent on clinical activities. ${ }^{[20]}$ The present study differs from previous studies in that it compares three levels of CPOE implementation rather than two, namely the present study compares a non-CPOE hospital, a short-term CPOE hospital, and a long-term CPOE hospital.

The objective of this study was to examine the effect of CPOE implementation on pharmacist workflow and time allotment on different categories of tasks, and specifically, to quantify and compare differences observed in the time spent on different task categories by pharmacists at hospitals with varying levels of implementation of a CPOE system.

\section{Methods}

A prospective time-and-motion study was designed to measure the time hospital pharmacists spent on different tasks. Hospital staff pharmacists were observed in the pharmacies of three community teaching hospitals within the same healthcare system in Houston, TX: Hospital A (non-CPOE system, 252 beds), Hospital B (short-term CPOE system, 274 beds), and Hospital C (long-term CPOE system, 142 beds). The data collection took place in two time periods, the first in April to May 2012, and the second from August to September 2012. There were no changes regarding CPOE implementation between these time periods for these three hospitals, and for both time periods, data was collected at all three hospitals. All three hospital pharmacies used the same information technology application, namely PharmNet ${ }^{\circledR}$ (Cerner Corporation, Kansas City, MO), within the Cerner Millenium ${ }^{\circledR}$ hospital-wide information system.

Within the Cerner system, the basic difference for pharmacists processing non-CPOE and CPOE orders is the transcription required for the non-CPOE orders; both types are still processed using the PharmNet ${ }^{\circledR}$ system. The sites were defined based on their predominant system and the length of the implementation. The CPOE sites still utilized some nonCPOE orders even after implementing CPOE, for instance Total Parenteral Nutrition orders were manually entered by the pharmacist into PharmNet ${ }^{\circledR}$. At the non-CPOE site, a small number of CPOE orders were processed because the Emergency Department was already using CPOE and prescriptions sent by the Emergency Department were processed as CPOE orders. Hospital $\mathrm{C}$ had been using CPOE for over five years prior to the start of the first observation period and thus was classified as long-term CPOE. Hospital B had adopted the CPOE system 19 months prior to the first observation period and was classified as short-term CPOE. The amount of time the CPOE system was implemented provided an opportunity to also consider the role it played and its effect on pharmacists' workflow.

The sample population consisted of order entry pharmacists 
at these locations, who worked in the central pharmacy for the hospital; other pharmacists were excluded. The sampling units were time (in minutes), collected in one-hour blocks. The pharmacists' activities were divided into 38 unique tasks (see Table 1), comprised of four general categories: clinical, distributive, administrative, and miscellaneous. The general categories were based on a list of four major activities (clinical, distributive, administrative, and miscellaneous) performed by hospital pharmacists, from a study published by Hatfield et al. in 2014. ${ }^{[20]}$ The list used by Hatfield et al. was refined from categories outlined in a study published by Gupta et al. in 2006 after consultation with pharmacy management and clinical pharmacists. ${ }^{[20-22]}$ The distributive category was broken down into three sub-categories: order entry, order verification, and other distributive task. The task list used by the Hatfield's study was modified for the present study to capture additional tasks which were observed during the pilot phase of the data collection tool.

Table 1. Tasks collected by the instrument, listed by activity

\begin{tabular}{llll}
\hline Task & Activity & Task & Activity \\
\hline 1. Clinical Interview & Clinical & 20. TPN Mixing Check & Distributive \\
2. Physician Order Form & Clinical & 21. Technician Check - non-IV room & Distributive \\
3. Rounds & Clinical & 22. Technician Check - IV room & Distributive \\
4. Patient Consult - Warfarin & Clinical & 23. Medication Prep/Delivery & Distributive \\
5. Patient Consult - Discharge & Clinical & 24. Chemo Mixing Check & Distributive \\
6. Direct Patient Care & Clinical & 25. Consult Pharmacist - Distributive & Distributive \\
7. Drug Information & Clinical & 26. Consult Technician & Distributive \\
8. E-Mar/Lab Review & Clinical & 27. Other - Distributive & Distributive \\
9. Consult Pharmacist - Clinical & Clinical & 28. Meeting & Admin. \\
10. Other - Clinical & Clinical & 29. Other - Administration & Admin. \\
11. Order Entry & Distributive & 30. Shift Report & Admin. \\
12. Chemo Order Review & Distributive & 31. Weekly personnel meeting & Admin. \\
13.TPN Order Review & Distributive & 32. Documentation & Admin. \\
14. Order Verification & Distributive & 33. Teaching/Mentoring & Admin. \\
15. IT Support & Distributive & 34. Emails & Admin. \\
16. Order Review and Order Entry for Surgery Patient & Distributive & 35. Q \& A - Management & Admin. \\
17. Pyxis Cart Fill Check & Distributive & 36. Scheduling & Admin. \\
18. Clarification - Nurse & Distributive & 37. Miscellaneous & Personal \\
19. Clarification - Physician & Distributive & & \\
\hline
\end{tabular}

A summary of the tasks measured can be viewed in Table 1. Tasks 1 through 10 were included in the clinical activity category and include such tasks as researching drug information, patient consultation upon discharge and performing a clinical interview. Tasks 11 through 27 were considered to be distributive and included all tasks that would be considered distributive in nature such as order entry, order verification, clarifying order, and medication prep. Tasks 28 through 37 were the tasks considered to be administrative in nature, including meeting, scheduling, and teaching. Task 38 was miscellaneous and included pharmacist time spent on personal activities and was not used in the analysis. The distributive tasks are further broken down into order verification, order entry, and other distributive tasks. The distributive category was defined to include all tasks associated with order entry and order verification, including discussions with personnel related to the distribution of medicines. The clinical category includes all tasks which are unambiguously clinical in nature, relating to the treatment of patients. The administrative cate-

Published by Sciedu Press gory was considered to be tasks that were neither distributive nor clinical, but were still value-filled tasks.

Order entry was defined as manual entry of medication orders from written or verbal communication and was considered to be a non-CPOE order. Hand-written orders were entered manually into PharmNet ${ }^{\circledR}$ by the pharmacists. Order verification was defined to be actions performed on orders received through PharmNet ${ }^{\circledR}$ systems and considered to be a CPOE order. CPOE orders were entered directly by the provider and sent for pharmacist verification through the PharmNet ${ }^{\circledR}$ system. The "other distributive tasks" category was defined to be distributive tasks not specifically associated with $\mathrm{CPOE}$ or non-CPOE orders.

\subsection{Data collection}

All data was collected by a single individual in order to reduce bias. The instrument used for data collection was an Access ${ }^{\circledR}$ (Microsoft Corp., Redmond, WA) database, 
originally developed by Partners Healthcare System for the AHRQ. ${ }^{[23]}$ The instrument was designed to capture time and motion data and was further modified for this specific study. One randomly selected pharmacist was observed at each data collection period, and observations were made in one-hour intervals. For each data block included, both hospital and pharmacist characteristics were obtained. A pilot study was conducted in order for pharmacists to become comfortable with the data collector, and the data collector's data collection was validated by a pharmacy manager expert with previous experience with the data collection tool.

Sample and site characteristics measured include the number of unique pharmacists observed at each site, pharmacist gender, pharmacist experience, the number of unique days data was collected at the site, and the level of CPOE implementation for the site. The number of unique pharmacists observed at each site was recorded because the lower number of unique pharmacists for one site could mean lower variation in observations for that site. Also, for each observation, the institutional experience of the pharmacist was recorded because it was possible that there could have been a significant difference in workflow productivity based on pharmacist experience.

Each hospital site was compared to the other two sites for differences in pharmacist time spent on activities: clinical, administrative, and distributive, as well as the subcategories for distributive, and the number of tasks performed by the pharmacist in an hour. The frequency of task changes was also collected and is defined to be the number of separate tasks recorded for the pharmacists in a given hour.

Data collection took place on weekdays in one-hour periods from 7:00 a.m. to 5:00 p.m., and the data collection was predetermined based on three criteria, including that the observation period would be considered to consist of non-irregular pharmacy workload, that it was possible to observe multiple pharmacists, and that the time period may span a minimum of four hours and a maximum of 10. Each one-hour block had to include at least $75 \%$ of time spent on non-miscellaneous tasks so that blocks in which the pharmacist took a large amount of personal time, such as a lunch break, would not be included. The data collector was trained to be as unobtrusive as possible. Also, the data collector had an opportunity to collect pilot data to reduce the effect of data collector bias. Further, the familiarity of the data collector with the pharmacists reduced possible bias due to the Hawthorne effect, and the pharmacists were not aware when the data collection was initiated for each observation period. In the event that multiple tasks were being completed at the same time, the data collector would make a judgment call of which task was occupying more of the pharmacist's attention.

This project was approved by the individual hospital administrators and the University of Houston's Division of Research Committee for the Protection of Human Subjects. Written approval was also granted by an authorized Systems Executive representing the Healthcare System, and consent forms were given to each pharmacist prior to requesting permission to include them in the study.

\subsection{Statistical analysis}

Data analysis was performed using SAS ${ }^{\circledR}$ version 9.3 (SAS Institute Inc., Cary, NC). Chi-square analyses were used to test for significant differences in the sample and site characteristics. Analysis of variance tests (ANOVAs) were used to test for significance between the test sites for time spent by pharmacists on different activities, and Tukey's range test was used for direct comparison between the sites. Additionally, comparisons were made between the sites by the total number of task changes by pharmacists per hour. The average different individual tasks per hour was calculated for each site and compared using ANOVA, along with Tukey's range test for post-hoc analysis. Apriori statistical significance was set at a level of 0.05 .

\section{RESULTS}

Data collection spanned from April 2, 2012, to October 2, 2012, where 252 hours of data were collected, of which, 235 hours met the inclusion criteria for analyses, and the most common reason an hour was excluded was due to the unanticipated absence of the pharmacist being observed. Sample and site characteristics are displayed in Table 2. The gender distribution, which represents the gender of the pharmacist for each individual data point indicates there was not a significant variation among the sites in terms of gender. There was a significant difference in pharmacist experience between the sites.

The three hospitals were compared by average time $(\mathrm{min} / \mathrm{hr})$ spent by pharmacists on each activity category (Table 3 shows the results of an ANOVA analysis). For every activity except for distributive tasks (combined), the ANOVA analysis indicated a statistically significant difference among the sites.

For the clinical activity, pharmacists at the non-CPOE site spent a statistically significant greater amount of time in clinical activities compared to the long-term CPOE site. For the administrative category, the difference in time spent per hour was not statistically significant in direct comparison of the sites. For order entry, there was a significant difference 
between all three sites, and for order verification, there was a significant difference measured between the non-CPOE site and the two CPOE sites. For the remaining distributive category, a significant difference was indicated between the long-term CPOE site when compared to the non-CPOE site and the short-term CPOE site.
The pharmacists at the non-CPOE site had a statistically significant number of fewer task changes per hour compared to the CPOE sites. For the non-CPOE site, short-term CPOE site, and long-term CPOE site, respectively, the average number of tasks changes per hour were: (mean $\pm S D)(23.74 \pm$ $7.32),(34.22 \pm 9.13)$, and (34.37 \pm 12.82$)$.

Table 2. Sample characteristics by hospital

\begin{tabular}{|c|c|c|c|c|}
\hline Hospital Characteristics & A: Non-CPOE & B: Short-term CPOE & C: Long-term CPOE & $p$-value \\
\hline Number of Unique Days Data Was Collected & 9 & 9 & 9 & - \\
\hline Number of Unique Pharmacists in Study & 11 & 8 & 12 & - \\
\hline Total Hours of Data Recorded & 78 & 78 & 79 & - \\
\hline Pharmacist Gender $^{\varphi}$ & & & & .709 \\
\hline - Male n $(\%)$ & $19(24.36)$ & $15(19.23)$ & $16(20.25)$ & \\
\hline - Female n $(\%)$ & $59(75.64)$ & $63(80.77)$ & $63(79.75)$ & \\
\hline Pharmacist Institutional Experience $\varphi \S$ & & & & $<.001$ \\
\hline - 0 to 1 year n $(\%)$ & $2(2.56)$ & $4(5.13)$ & $0(0.0)$ & \\
\hline - 1 to 10 years $\mathrm{n}(\%)$ & $32(41.03)$ & $69(88.46)$ & $63(79.75)$ & \\
\hline - $10+$ years $\mathrm{n}(\%)$ & $44(56.41)$ & $5(6.41)$ & $16(20.25)$ & \\
\hline
\end{tabular}

Note. CPOE = computerized provider order entry; ${ }^{*}$ numerical $p$-values calculated using ANOVA, categorical, chisquare; $p$-value of $<.05$ is considered statistically significant; ${ }^{\varphi}$ numbers are based on the aggregate results from each 1 hour block of collected data; ${ }^{8}$ chisquare analysis significant for each comparison: $\mathrm{a}$ to $\mathrm{b}$, a to $\mathrm{c}$, and $\mathrm{b}$ to $\mathrm{c}$

Table 3. Average time (min/hr) spent by hospital staff pharmacists on activities

\begin{tabular}{lllll}
\hline \multirow{2}{*}{ Activity } & \multicolumn{3}{c}{ Mean $\pm \boldsymbol{S D}$} \\
\cline { 2 - 4 } & A: Non-CPOE Hospital & B: Short-term CPOE Hospital & C: Long-term CPOE Hospital & \multicolumn{2}{c}{-value $^{*}$} \\
\hline Clinical $^{\varphi}$ & $6.55 \pm 6.40$ & $4.95 \pm 4.15$ & $3.79 \pm 4.91$ & .0046 \\
Administrative $^{\S}$ & $5.55 \pm 6.76$ & $5.59 \pm 6.04$ & $8.15 \pm 8.31$ & .0337 \\
Order Entry $^{\text {Order Verification }}{ }^{\#}$ & $29.62 \pm 11.24$ & $17.44 \pm 10.73$ & $10.27 \pm 8.88$ & $<.0001$ \\
All Other Distributive Tasks $^{\delta}$ & $0.88 \pm 1.77$ & $13.93 \pm 8.50$ & $16.60 \pm 9.63$ & $<.0001$ \\
Distributive Tasks (Combined) $^{\epsilon}$ & $13.60 \pm 10.04$ & $15.86 \pm 8.38$ & $19.66 \pm 8.42$ & .0002 \\
Miscellaneous $^{\epsilon}$ & $3.78 \pm 4.64$ & $47.23 \pm 8.43$ & $46.53 \pm 9.17$ & .0850 \\
\hline
\end{tabular}

Note. $\mathrm{CPOE}=$ computerized provider order entry; Minutes may not equal to exactly 60 due to rounding to second digit after decimal; ${ }^{*} p$-value calculated by ANOVA - a value of $<.05$ is considered statistically significant; ${ }^{\phi}$ Tukey's test performed to test statistical significance between each site; A to C was significant; ${ }^{\S}$ Tukey's test performed to test statistical significance between each site; no direct comparisons were significant; ${ }^{\&}$ Tukey's test performed to test statistical significance between each site; A to B, A to C, and B to C were all significant; ${ }^{\#}$ Tukey's test performed to test statistical significance between each site; A to B and A to $\mathrm{C}$ were significant; ${ }^{\delta}$ Tukey's test performed to test statistical significance between each site; A to C and B to C was significant; ${ }^{\epsilon}$ Tukey's test performed to test statistical significance between each site; A to B and A to C was significant

\section{Discussion}

The pharmacists at both the short-term and long-term-CPOE sites spent more time on distributive tasks and less time on clinical tasks compared to the non-CPOE site, and a particular result of note was that pharmacists at the long-term CPOE site spent a statistically significant less amount of time on clinical tasks compared to the non-CPOE site. These results are unexpected and opposite to those of the previously mentioned study published by Hatfield et al., in which it was found that CPOE implementation was associated with a decrease in the amount of time pharmacists spent on distributive activities and increase the amount of time spent on clinical activities. ${ }^{[20]}$ One possibility to explain the differ- ence is that the previous study was conducted in the span of two weeks, with only a total of 48 observations, and included 11 different pharmacists compared to this study which was conducted during two different time periods of the year, had 238 observations, with 31 different pharmacists, allowing a greater depth and variety of data collection. In addition, factors that were not measured, such as number of orders requested, could have significantly differed among the hospitals. If the pharmacists completed a larger number of order requests, this could account for the study results. Number of orders requested was not collected because permission was not given by the hospital system.

A particular facet of the results is that while time spent on 
clinical activities at the long-term CPOE site did not differ significantly compared to the short-term CPOE site, there is a noticeable trend of decreasing time spent on clinical activities from the non-CPOE site to the short-term CPOE site to the long-term CPOE site. This trend raises questions if the amount of time pharmacists spend doing clinical activities could decrease with the depth of CPOE implementation. A similar time-and-motion study was published by Westbrook et al. ${ }^{[24]}$ in 2013 that measured how introduction of an electronic medication management system (eMMS) affected the amount of time doctors and nurses spent on direct patient care and medication tasks, and it was concluded that the introduction of eMMS did not redistribute time spent on medication tasks or direct patient care.

One possible explanation for the results of the present study is that utilization of CPOE places new burdens and challenges on the pharmacists, with the electronic system directly or indirectly requiring more of the pharmacists' time. A study recently published by Sinsky et. al., ${ }^{[25]}$ indicated that physicians are spending an alarming amount of time on electronic health records (EHR) and deskwork. The study found that physicians were spending over twice the amount of time on EHR and desk work than on direct clinical face time. It is possible a similar burden of electronic systems is being placed on the pharmacists. Previous studies have shown that while implementing CPOE can improve the safety and efficiency of order processing, it can create new challenges and resource demands that place increased burden on pharmacists, such as increasing new types of errors and problems caused by CPOE systems. ${ }^{[26-29]}$ While CPOE systems can increase efficiency in many areas, such systems should be monitored by management so any new problems that arise can be addressed.

A similar time-and-motion study was published by Lo et al. in 2010 that measured time spent on tasks, task frequencies, and other comparisons of tasks among pharmacists in a hospital ward with eMMS compared to a hospital ward without eMMS. They found that pharmacists' work patterns varied significantly and attributed the changes to increased access to speedy and easy information retrieval for reviews and improved clarity regarding orders due to the presence of eMMS. ${ }^{[30]}$ This factor could have played a part in the present study because in the present study, the CPOE sites had a significantly larger number of task changes per hour compared to the non-CPOE site. Because of this, even though the number of minutes spent on clinical tasks per hour decreased, the efficiency of clinical related tasks could have been increased, more than compensating for time loss.

The frequency of task changes is of interest because it could indicate potential differences in workflow. Pharmacists at both of the CPOE sites were recorded to have an average of approximately ten more task changes per hour compared to the non-CPOE site. This is a statistically significant result that could mean a major shift in workflow dynamics when comparing a non-CPOE system versus a CPOE system. This difference could indicate an increased pace in the workflow or an increased productivity for the pharmacies that implemented CPOE, regardless of if the CPOE system has been implemented for 19 months or longer. The significant difference in amount of time pharmacists spent on the miscellaneous category (personal time) in sites with CPOE implementation compared to the non-CPOE site could again indicate a fundamental change of workflow or work environment following CPOE implementation.

Comparing the short-term and long-term CPOE sites, two of the activity categories compared were significantly different (Order Entry and all other distributive tasks). This indicates that time elapsed following CPOE implementation may have an effect on pharmacists' task time distribution. Comparing the non-CPOE site, short-term site, and long-term site as a continuum, four trends arise: Less time spent on clinical activities, less time spent on order entry, more time spent on order verification, and more time spent on all other distributive tasks. Even though the difference in the non-CPOE site to the others in terms of order entry and order verification discrepancy is expected, the differences between the shortterm and long-term CPOE sites indicate that the long-term CPOE site has an increased depth of CPOE implementation. The results suggest that after initial CPOE implementation, the workflow and productivity in the pharmacy continues to change.

The pharmacist characteristics were comparable across the three sites, with pharmacist experience having the most variance. Hospital A had a greater proportion of pharmacists that had 10 or more years of experience, and there is a possibility this discrepancy in pharmacy experience could have had an effect on their time allotment on different tasks. It was also noted that in many ways the pharmacies differed ostensibly in many aspects that were not measured, including, the unique needs of the hospital, the pharmacy manager's managing style, the physical layout of the pharmacies, and individual pharmacist work ethic.

While previous studies have shown the implementation of a CPOE system can improve patient safety, there are still possibilities of unintended consequences, in which the mechanisms are not fully understood. Management should consider putting in place metrics to assess workflow and productivity prior to technological implementations and continue moni- 
toring even after the implementation is complete. A potential topic of future interest, not addressed by this study is if the pharmacist time spent in the distributive category corresponds to a greater number of orders being completed by the pharmacists. This would be very important to determining some of the workflow and productivity changes caused by implementation of a CPOE system. In addition, future studies should investigate if CPOE systems do actually cause less time to be spent on clinical work, such a study could specifically look at how implementation of CPOE affects the pharmacists' time spent on clinical activity, and if in the presence of a CPOE system, more pressure is placed on pharmacists to spend more time on filling orders and other distributive tasks.

\section{Strengths and limitations}

There are some tasks by the pharmacists which could not be observed, including decision-making and personal judgment. Data collection was based on observable actions. In addition, tasks were considered to be clinical only if they were unambiguous and clearly clinical in nature, and it is possible additional tasks could be classified as clinical such as consulting with a nurse about a particular patient's medicine or review of a particular chemotherapy order. The generalizability of the study is limited in that this study included only a single hospital system and hospital pharmacies and CPOE systems can vary greatly. The validity of the comparisons of the hospital sites may be questioned because the dynamic of pharmacist workflow may be influenced by individual pharmacy managers, needs of the hospital, and other factors not measured. Another noted limitation of the study is that the site considered to be non-CPOE did have a small number of $\mathrm{CPOE}$ orders processed. The results showed that pharmacists at the non-CPOE site spent on average 0.88 minutes per hour on CPOE order verification compared to 29.62 average minutes spent on non-CPOE order entry, so the bias introduced by the small number of CPOE orders in the non-CPOE site should be small. Other biases that could have affected the study include observer bias, and bias from the Hawthorne effect. However, the Hawthorne effect was should have been limited because the sites of this study regularly host multiple $3^{\text {rd }}$ and $4^{\text {th }}$-year pharmacy students, so the pharmacists should have been accustomed to being observed.

A major limitation is the unavailability of the number of orders the pharmacists completed. This information could help explain possible workflow and productivity differences between the short-term CPOE, long-term CPOE, and nonCPOE systems.

The sample size of 235 observations provides the study with a large enough sample size to answer the main objective questions, and observer bias was limited because a single observer was used for each of the three different sites.

\section{Conclusions}

At the CPOE pharmacies, less time was spent on order entry; however, the amount of time gained by spending less time on order entries was more than made up for in the amount of time spent doing order verification tasks. Pharmacists at the CPOE hospitals spent more time on distributive tasks and less time on clinical activities. Additionally, while the benefits of implementing a CPOE system are many, the presence of a CPOE system as well as time elapsed since CPOE implementation could have a dramatic effect on pharmacist workflow dynamics and time spent on certain activities. CPOE systems should be closely monitored by pharmacy managers to ensure workflow remains efficient and factors such as time spent on clinical tasks are not negatively affected.

\section{ACKNOWLEDGements}

The authors are grateful to the directors of pharmacy and hospital administrators who granted approval for the study and to the pharmacists at the hospitals for their help in the successful completion of the study.

\section{CONFlicts OF INTEREST Disclosure}

The authors declare they have no conflicts of interest.

\section{REFERENCES}

[1] Bates DW, Teich JM, Lee J, et al. The impact of computerized physician order entry on medication error prevention. Journal of the American Medical Informatics Association. 1999; 6(4): 313-21. http://www.ncbi.nlm.nih.gov/pmc/articles/PMC61372/

[2] Devine EB, Hansen RN, Wilson-Norton JL, et al. The impact of computerized provider order entry on medication errors in a multispecialty group practice. Journal of the American Medical Informatics Association. 2010; 17(1): 78-84. PMid: 20064806. https://doi.org/10.1197/jamia.M3285
[3] Hunt DL, Haynes R, Hanna SE, et al. Effects of computer-based clinical decision support systems on physician performance and patient outcomes: A systematic review. JAMA. 1998; 280(15): 1339-46. https://doi.org/10.1001/jama.280.15.1339

[4] Kawamoto K, Houlihan CA, Balas EA, et al. Improving clinical practice using clinical decision support systems: A systematic review of trials to identify features critical to success. BMJ: British Medical Journal. 2005; 330(7494): 765-65. https://doi.org/10.1136/ bmj.38398.500764.8F

[5] Leapfrog Group. Factsheet: Computerized physician order entry. 
Washington, DC; Revised April 2016 [cited 2017 Oct. 6]. Available from: http://www. leapfroggroup.org/sites/default/ files/Files/CPOE\%20Fact\%20Sheet.pdf

[6] Leapfrog Group. Factsheet: Computerized physician order entry. Washington, DC. Revised April 2016 [cited 2017 Oct 6]. Available from: http://www. leapfroggroup.org/sites/default/ files/Files/CPOE\%20Fact\%20Sheet.pdf

[7] Centers for Medicare and Medicaid (CMS). Medicaid Services Stage 2 eligible professional meaningful use core measures: measure 1 of 17. Woodlawn, MD; 2012 [cited 2017 Oct 6] Available from: www. cms.gov

[8] Office of the National Coordinator for Health IT U.S. Hospital Adoption of Computerized Capabilities to Meet Meaningful Use Stage 2 Objectives, Health IT Quick-Stat \#23. Washingon, DC; 2014 [cited 2017 Oct 6]. Available from: http://dashboard.healthit.gov /quickstats/quickstats.php

[9] Kuperman GJ, Gibson RF. Computer physician order entry: Benefits, costs, and issues. Annals of Internal Medicine. 2003; 139(1) 31-39. https://doi .org/10.7326/0003-4819-139-1-20030 7010-00010

[10] Niazkhani Z, Pirnejad H, Berg M, et al. The impact of computerized provider order entry systems on inpatient clinical workflow: A literature review. Journal of the American Medical Informatics Association 2009; 16(4): 539-49. https ://doi.org/10.1197/jamia.M2419

[11] Pitre M, Ong K, Huh JH. Thorough planning and full participation by pharmacists is key to moe/mar success. Healthcare Quarterly. 2006; 10(Sp): 43-49. https://doi .org/10.12927/hcq. . 18496

[12] Davidson EJ, Chismar W. Examining the organizational implications of it use in hospital-based health care: A case study of computerized order entry. Proceedings of the 32nd Annual Hawaii International Conference on Systems Sciences; Dept. of Decisions Sci., Hawaii Univ., Honolulu, HI, USA; 1999 Jan 5-8 ( Systems Sciences, 1999. HICSS-32). Available from: https://www. computer.org/csd 1/proceedings/hicss/1999/0001/04/00014008.pdf

[13] Jensen JR. The effects of computerized provider order entry on medication turn-around time: A time-to-first dose study at the providence portland medical center. AMIA Annual Symposium Proceedings. 2006; 2006: 384-88.

[14] Zamora N, Carter M, Saull-Mccaig S. The benefits of the moe/mar implementation: A quantitative approach. Healthcare Quarterly. 2006; 10(Sp): 77-83. https://doi .org/10.12927/hcq. . 18505

[15] Lehman ML, Brill JH, Skarulis PC, et al. Physician order entry impact on drug turn-around times. Proceedings of the AMIA Symposium. 2001; 359-63. https://www.ncbi.nlm.nih.gov/pmc/article s/PMC2243330/

[16] Taylor R, Manzo J, Sinnett M. Quantifying value for physician order-entry systems: A balance of cost and quality. Healthcare Financial Management. 2002; 56(7): 44-8. PMid: 12119829. Available from: https://healthit.ahrq.gov/health-it-t ools-and-resources/health-it-bibliography/return-i nvestment/quantifying-value-physician

[17] Mekhjian HS, Kumar RR, Kuehn L, et al. Immediate benefits realized following implementation of physician order entry at an academic medical center. Journal of the American Medical Informatics Association. 2002; 9(5): 529-39. https://doi.org/10.1197/jamia. M1038

[18] Cordero L, Kuehn L, Kumar RR, et al. Impact of computerized physician order entry on clinical practice in a newborn intensive care unit. J Perinatol. 2000; 24(2): 88-93. https ://doi .org/10.1038/sj .jp. 7211000

[19] Bhosle M, Sansgiry SS. Computerized physician order entry systems: Is the pharmacist's role justified? Journal of the American Medical Informatics Association. 2004; 11(2): 125-26. https : //doi.org/10.1197/jamia.M1469

[20] Hatfield MD, Cox R, Mhatre SK, et al. Impact of computerized provider order entry on pharmacist productivity. Hospital Pharmacy. 2014; 49(5): 458-65. PMid: 24958959. https ://doi.org/10.1 310/hpj 4905-458

[21] Gupta SR, Wojtynek JE, Walton SM, et al. Monitoring of pharmacy staffing, workload, and productivity in community hospitals. American Journal of Health-System Pharmacy. 2006; 63(18): 1728-34 https://doi.org/10.2146/ajhp060012

[22] Cox R. Impact of Computerized Physician Order Entry on Pharmacy Productivity; Time and Motion Study. [thesis] [Houston (TX)]: University of Houston; 2011.

[23] Partners Healthcare System. Time and motion database. Last revised 2013[cited Oct. 6 2013]. Available from: https://innovations . ahrq.gov/qualitytools/time-and-motion-database

[24] Westbrook JI, Li L, Georgiou A, et al. Impact of an electronic medication management system on hospital doctors' and nurses' work: a controlled pre-post, time and motion study. Journal of the American Medical Informatics Association: JAMIA. 2013; 20(6): 1150-1158. https://doi:10.1136/amiajnl-2012-001414

[25] Sinsky C, Colligan L, Li L, et al. Allocation of Physician Time in Ambulatory Practice: A Time and Motion Study in 4 Specialties. Ann Intern Med. 2016; 165: 753-760. https ://doi : 10.7326/M16-0961

[26] McMullen CK, Macey TA, Pope J, et al. Effect of computerized prescriber order entry on pharmacy: Experience of one health system. American Journal of Health-System Pharmacy. 2015; 72(2): 133-42. https://doi.org/10.2146/ajhp140106

[27] Weir CR, McCarthy CA. Using implementation safety indicators for cpoe implementation. Jt Comm J Qual Patient Saf. 2009; 35: 21-28. https://doi.org/10.1016/S1553-7250(09) 35004-7

[28] Estellat C, Colombet I, Vautier S, et al. Impact of pharmacy validation in a computerized physician order entry context. International Journal for Quality in Health Care. 2007; 19(5): 317-25. https://doi.org/10.1093/intqhc/mzm025

[29] Magid S, Forrer C, Shaha S. Duplicate orders: An unintended consequence of computerized provider/physician order entry (cpoe) implementation: Analysis and mitigation strategies. Applied Clinical Informatics. 2012; 3(4): 377-91. https ://doi .org/10.4338/AC I-2012-01-RA-0002

[30] Lo C, Burke R, Westbrook JI. Electronic medication management systems' influence on hospital pharmacists' work patterns. Journal of Pharmacy Practice and Research. 2010; 40(2): 106-10. https://doi.org/10.1002/j.2055-2335.2010.tb00516.x 